\title{
Nearly White-Light Emission From GaN-Based Light-Emitting Diodes Integrated With a Porous $\mathrm{SiO}_{2}$ Layer
}

\author{
Chih-Hao Hsieh, Min-Yung Ke, Ghien-An Shih, Tzu-Yang Chiu, and Jian Jang Huang
}

\begin{abstract}
In this letter, we develop a nearly white-light-emitting device by integrating blue/green emission from a GaN-based lightemitting diode with red emission from a porous $\mathrm{SiO}_{2}$ layer. The porous $\mathrm{SiO}_{2}$ layer was fabricated by a novel process procedure to create $\mathrm{Si}$ nanocrystals on top of the n-type GaN layer. Red light is generated from the metal-oxide-semiconductor $\left(\mathrm{Ni}-\mathrm{Au}-\mathrm{SiO}_{2}\right.$ oxide-n-type GaN) structure due to the electron-hole recombination in the Si nanocrystals. The device shows a blue light emission at a low biased voltage and nearly white-light emission (green and red colors) at a bias voltage between 14 and $16 \mathrm{~V}$. Our results show the potential of applying such an integrated structure to white-light illumination.
\end{abstract}

Index Terms-Light-emitting diodes (LEDs), Si-nanocrystal, $\mathrm{SiO}_{2}$ porous layer, white light.

\section{INTRODUCTION}

W HITE-LIGHT-EMITTING diodes (LEDs) have become popular due to their applications to backlights for thin-film transistor-liquid crystal displays and general lighting. Typically, they can be fabricated with the combination of discrete red, green, and blue LEDs [1]. The phosphor converted LED is an alternative approach for white-light illumination [2]. They can be realized by mixing blue light from GaN-based LEDs with yellow light converted from the blue pumped phosphor, or by mixing green, red, and blue colors converted from ultraviolet pumped phosphors [3]. Despite the availability of choices of using phosphor species for selected wavelength conversion, there are disadvantages of phosphor converted LEDs. For example, the conversion efficiency of phosphor species is typically very low. This poor energy conversion is due to a Stokes energy loss when converting short-wavelength photons to long wavelength ones. Also, the phosphor converted LEDs do not allow for the extensive tunability, particularly in terms of spectral modulation [1]. In light of those drawbacks, direct electron-hole recombination or direct optical pump semiconductor materials are alternate candidates for high efficient light sources.

Manuscript received June 23, 2006; revised December 13, 2006. This work was supported by the National Science Council, Taiwan, under Grant NSC 94-2215-E-002-028.

The authors are with the Graduate Institute of Electro-Optical Engineering and Department of Electrical Engineering, National Taiwan University, Taipei, 106 Taiwan, R.O.C. (e-mail: jjhuang@cc.ee.ntu.edu.tw).

Digital Object Identifier 10.1109/LPT.2007.893760
Silicon nanocrystals (nc-Si) embedded in $\mathrm{SiO}_{2}$ matrix (nc-Si-SiO ${ }_{2}$ ) is attracting a lot of interest in its efficiency and stability of light emission at room temperature. Various technologies have been proposed to fabricate nc-Si structures, such as plasma-enhanced chemical vapor deposition (PECVD) [4], electron-beam evaporation [5], and Si-ion implantation [6]. Photoluminescence (PL) spectra from the blue to near-infrared region were demonstrated [7]-[9]. Even though it is generally thought that the quantum confinement effect is one of the main mechanisms for light emission, point defects, such as from Si-ion-implanted $\mathrm{SiO}_{2}$, are also considered to be another source for light emissions [10]. In addition, electroluminescence (EL) spectra of the metal-oxide-semiconductor (MOS) structures (where the material nc-Si-SiO ${ }_{2}$ or $\mathrm{Si}$-ion implanted $\mathrm{SiO}_{2}$ is regarded as the oxide layer and $\mathrm{Si}$ substrate as the semiconductor) were also reported with turn-on voltages between 3.3 and $86 \mathrm{~V}$ in the 700 900 nm wavelength range [10]-[12].

In this letter, we develop a novel integrated white-light-emitting device. By combining the nc-Si MOS with the GaN-based LED structure, i.e., by depositing a porous $\mathrm{SiO}_{2}$ layer on top of the GaN-based LED, nearly white-light emission with dual spectrum peaks at 496.4 and $828.6 \mathrm{~nm}$ are demonstrated at room temperature. The turn-on voltage of the nc-Si MOS device is as low as $13.7 \mathrm{~V}$ and the operation voltage of the nearly white-light MOS-LED device is between 14 and $16 \mathrm{~V}$. The MOS-LED structure shows the potential of applying such an integrated device to white-light generation.

\section{Material Growth And Device Fabrication}

The GaN-based LED sample with a PL peak at around $480 \mathrm{~nm}$ was grown on a c-plane sapphire substrate by metal-organic chemical vapor deposition. The material structure is composed of a $25-\mathrm{nm}$ GaN buffer layer, a 1.8- $\mu \mathrm{m}$ Si-doped n-type GaN layer, a ten-period InGaN-GaN multiple quantum-well structure, and a 200-nm p-type $\mathrm{GaN}$ layer. The patterned $\mathrm{SiO}_{2}$ layer was deposited on top of the GaN n-type layer by PECVD with an initial thickness of $340 \mathrm{~nm}$. We then wet-etched the $\mathrm{SiO}_{2}$ layer by buffer oxide etcher $\left(\mathrm{NH}_{4} \mathrm{~F}: \mathrm{HF}=6: 1\right)$ to thin down the $\mathrm{SiO}_{2}$ layer. In the subsequent step, the sample was dry etched by inductively coupled plasma reactive ion etching to create porous profile on $\mathrm{SiO}_{2}$ layer. We used $\mathrm{Ar}, \mathrm{SiCl}_{4}$ and $\mathrm{Cl}_{4}$ as etching gases with a flow rate 20,1 , and $15 \mathrm{sccm}$, respectively. The $\mathrm{SiO}_{2}$ layer thickness was optimized by considering both the generated output optical power from the $\mathrm{SiO}_{2}$ layer (nc-Si) and the operating voltage. Typically, a thicker $\mathrm{SiO}_{2}$ layer is preferred for luminescence which, however, requires a 


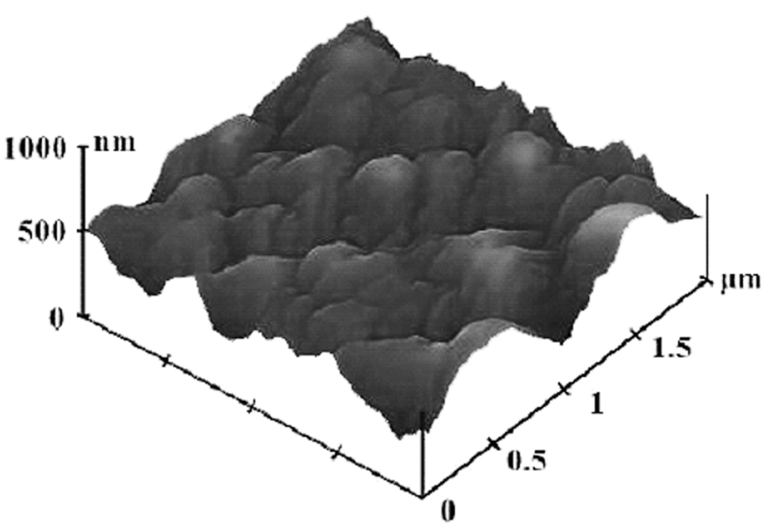

Fig. 1. AFM surface profile of the porous $\mathrm{SiO}_{2}$ layer.

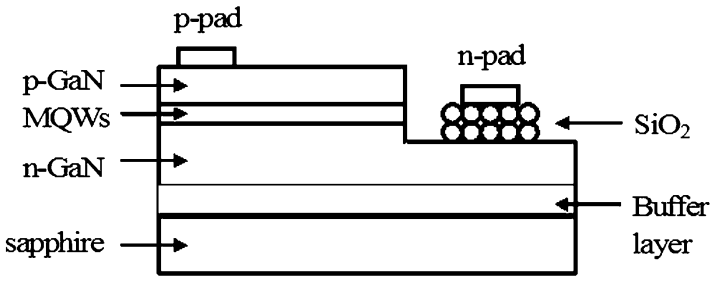

Fig. 2. Schematic illustration of a GaN-based LED integrated with a porous $\mathrm{SiO}_{2}$ layer.

higher biased voltage. In our case, the optimum thickness of the porous $\mathrm{SiO}_{2}$ layer is around $200 \mathrm{~nm}$. The atomic force microscopic (AFM) surface profile of the porous $\mathrm{SiO}_{2}$ layer grown on top of the n-type GaN layer is shown in Fig. 1. The lateral diameter of nc-Si is approximately $500 \mathrm{~nm}$. In the next step, $\mathrm{Ni}-\mathrm{Au}$ metal layers were evaporated as the p-type electrode and were alloyed to obtain optimized ohmic contact condition. We then coated $\mathrm{Ti}-\mathrm{Au}$ directly on top of the porous $\mathrm{SiO}_{2}$ layer as the n-type contact. The $\mathrm{Ti}-\mathrm{Au}-\mathrm{SiO}_{2}-\mathrm{GaN}$ layer combination forms an MOS structure (see Fig. 2) and red emission can be observed after a $750{ }^{\circ} \mathrm{C}$ annealing in our experiment. The high-temperature annealing $\left(>700{ }^{\circ} \mathrm{C}\right)$ deactivates the neutral oxygen vacancies defect $(\mathrm{O} \equiv \mathrm{Si}-\mathrm{Si} \equiv \mathrm{O})$ in the porous $\mathrm{SiO}_{2}$ layer, and meanwhile generates nc-Si [13]. Electron-hole recombination can occur in the porous $\mathrm{SiO}_{2}$ layer (nc-Si).

\section{CHARACTERIZATIONS}

The EL spectra of our samples were taken at various bias voltages [see Fig. 3(a)] at room temperature. At a low bias voltage, electrons from the n-type electrode tunnel through the porous $\mathrm{SiO}_{2}$ layer and recombine with holes in the GaN-based active layer to generate blue emission. Therefore, the blue emission is dominated at low bias voltages. The peak wavelength is around $487.6 \mathrm{~nm}$ when biased at $14 \mathrm{~V}$. As we increase the applied voltage to $15 \mathrm{~V}$, red emission is observed as the carrier recombination in the MOS structure starts to occur. It is also noted that the relative intensity of the blue emission decreases as the applied voltage increases, which is attributed to the fact that more and more carriers are recombined in the MOS region when the bias voltage increases from 14 to $15 \mathrm{~V}$. The blue emission is further decreased as the applied voltage is increased to $16 \mathrm{~V}$. The peak wavelength of the GaN-based LED is shifted to $496.4 \mathrm{~nm}$
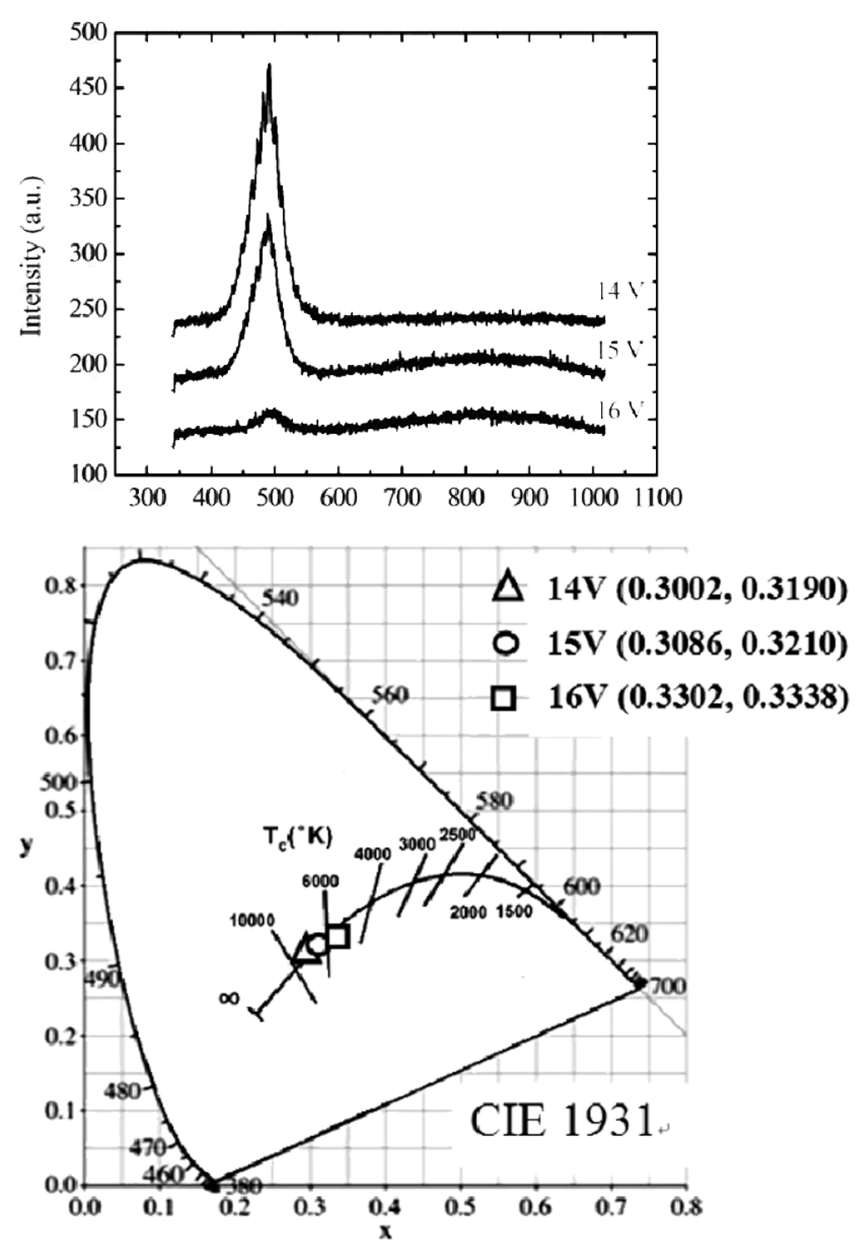

Fig. 3. (a) Room-temperature EL spectra and (b) CIE 1931 chromaticity diagram of the light emission of LEDs at bias voltages 14,15 , and $16 \mathrm{~V}$.

due to quantum confinement stark effect. The relative intensities of wavelength 496.4 and $828.6 \mathrm{~nm}$ have become comparable at the bias voltage 16 V. Fig. 3(b) shows the CIE 1931 chromaticity diagram with corresponding light-emitting colors at different bias voltages. With the increase of bias voltages, the bluish color ( $x=0.3002, y=0.3190)$ at $14 \mathrm{~V}$ turns to a nearly white color $(x=0.3302,0.3338)$ at $16 \mathrm{~V}$.

We further compare the current-voltage $(I-V)$ characteristic of the MOS-LED structure with that of the conventional LED sample (the one without any $\mathrm{SiO}_{2}$ layer coated). As shown in Fig. 4, the $I-V$ curve of the conventional LED shows a diode behavior (dashed line), while that of the MOS-LED structure acts like a normal LED in series with a resistor when the bias voltage is below $13.7 \mathrm{~V}$. Since carriers from the n-type electrode of the MOS-LED structure have to tunnel through the thin porous $\mathrm{SiO}_{2}$ layer, the effective current under the same bias voltage of the MOS-LED structure is smaller than that of the conventional LED structure. When the bias voltage is above $13.7 \mathrm{~V}$ (the threshold voltage of the MOS device), the device is turned on and electrons from the n-type electrode start to recombine with holes in the nc-Si. A negative resistance is observed at bias voltages above $13.7 \mathrm{~V}$, indicating the generation of red light. The negative resistance in Fig. 4 corresponds to the onset of red light emission in Fig. 3 and meanwhile indicates the decrease 


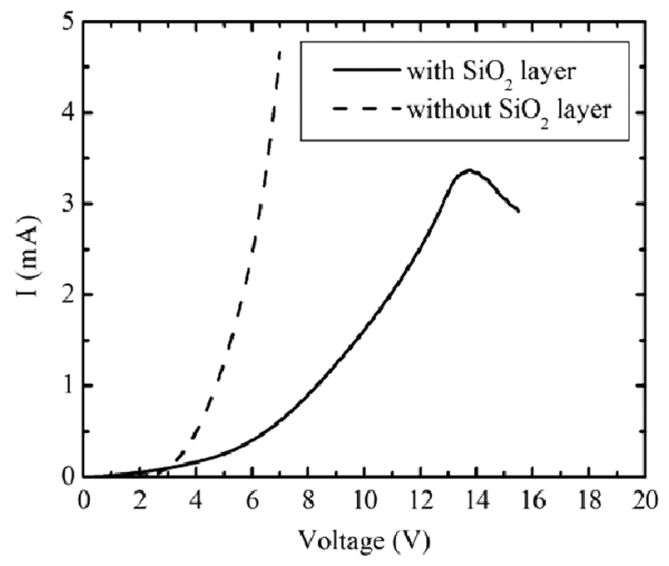

Fig. 4. $I-V$ curves of the MOS-LED sample (solid line) and the conventional LED (dashed line).

of blue intensity. The MOS structure breaks down (blows up) at bias voltages beyond $16 \mathrm{~V}$, which is due to large numbers of electrons tunneling through the porous $\mathrm{SiO}_{2}$ layer.

\section{CONCLUSION}

We have developed a novel integrated white-light-emitting device. By integrating the nc-Si MOS with the GaN-based LED structures, i.e., by depositing a porous $\mathrm{SiO}_{2}$ layer on top of the GaN-based LED, nearly white-light emission with dual spectrum peaks at 496.4 and $828.6 \mathrm{~nm}$ were demonstrated at room temperature. The turn-on voltage of the nc-Si MOS device is as low as $13.7 \mathrm{~V}$ and the operating voltage of the nearly white-light MOS-LED device is between 14 and $16 \mathrm{~V}$. In addition, a negative resistance is observed from the $I-V$ curve of the MOS-LED structure at a bias voltage above $13.7 \mathrm{~V}$. It is attributed to the fact that some carriers start to recombine in the MOS region. The relative intensity of red emission is thus increased while that of the blue emission is decreased. Our results indicate the potential of applying such an integrated structure to white-light generation.

\section{REFERENCES}

[1] E. F. Schubert and J. K. Kim, "Solid-state light sources getting smart," Science, vol. 308, no. 5726, pp. 1274-1278, May 27, 2005.

[2] H. Wu, X. Zhang, C. Guo, J. Xu, M. Wu, and Q. Su, "Three-band white light from InGaN-based blue LED chip precoated with green/red phosphors," IEEE Photon. Technol. Lett., vol. 17, no. 6, pp. 1160-1162, Jun. 2005.

[3] J. K. Sheu, S. J. Chang, C. H. Kuo, Y. K. Su, L. W. Wu, Y. C. Lin, W. C. Lai, J. M. Tsai, G. C. Chi, and R. K. Wu, "White-light emission from near UV InGaN-GaN LED chip precoated with blue/green/red phosphors," IEEE Photon. Technol. Lett., vol. 15, no. 1, pp. 18-20, Jan. 2003.

[4] D. Pacifici, E. C. Moreira, G. Franzò, V. Martorino, F. Priolo, and F. Iacona, "Defect production and annealing in ion-irradiated Si nanocrystals," Phys. Rev. B, vol. 65, p. 144109, Apr. 2002.

[5] Q.-Y. Ye, R. Tsu, and E. H. Nicollian, "Resonant tunneling via microcrystalline-silicon quantum confinement," Phys. Rev. B, vol. 44, no. 4, pp. 1806-1811, 1991.

[6] A. Pèrez-Rodrìguez, O. González-Varona, B. Garrido, P. Pellegrino, J. R. Morante, C. Bonafos, M. Carrada, and A. Claverie, "White luminescence from $\mathrm{Si}^{+}$and $\mathrm{C}^{+}$ion-implanted $\mathrm{SiO}_{2}$ films," J. Appl. Phys., vol. 94, no. 1, pp. 254-262, Jul. 2003.

[7] X. Zhao, O. Schoenfeld, J. Kusano, Y. Aoyagi, and T. Sugano, "Observation of direct transitions in silicon nanocrystallites," Jpn. J. Appl. Phys., vol. 33, no. 7A, pt. 2, pp. L899-L901, Jul. 1994.

[8] T. Shimizu-Iwayama, K. Fujita, S. Nakao, K. Saitoh, T. Fujita, and N. Itoh, "Visible photoluminescence in $\mathrm{Si}^{+}$-implanted silica glass," $J$. Appl. Phys., vol. 75, no. 12, pp. 7779-7783, Jun. 1994.

[9] P. Mutti, G. Ghislotti, S. Bertoni, L. Bonoldi, G. F. Cerofolini, L. Meda, E. Grilli, and M. Guzzi, "Room-temperature visible luminescence from silicon nanocrystals in silicon implanted $\mathrm{SiO}_{2}$ layers," Appl. Phys. Lett., vol. 6, pp. 851-853, Feb. 1995.

[10] G.-R. Lin, C.-J. Lin, C.-K. Lin, L.-J. Chou, and Y.-L. Chueh, "Oxygen defect and Si nanocrystal dependent white-light and near-infrared electroluminescence of Si-implanted and plasma-enhanced chemical-vapor deposition-grown Si-rich $\mathrm{SiO}_{2}$, J. Appl. Phys., vol. 97, pp. 094306-094306-8, Apr. 2005.

[11] G. Franzò, A. Irrera, E. C. Moreira, M. Miritello, F. Iacona, D. Sanfilippo, G. Di Stefano, P. G. Fallica, and F. Priolo, "Electroluminescence of silicon nanocrystals in MOS structures," Appl. Phys. A: Mater. Sci. Process., vol. 74, no. 1, pp. 1-5, Jan. 2002.

[12] J. Valenta, N. Lalic, and J. Linnros, "Electroluminescence microscopy and spectroscopy of silicon nanocrystals in thin $\mathrm{SiO}_{2}$ layers," Opt. Mater., vol. 17, no. 1-2, pp. 45-50, Jun. 2001.

[13] J. H. Son, T. G. Kim, S. W. Shin, H. B. Kim, W. S. Lee, S. Im, J. H. Song, C. N. Whang, and K. H. Chae, "Blue and red luminescence from $\mathrm{Si}$ ion-irradiated $\mathrm{SiO}_{2} / \mathrm{Si} / \mathrm{SiO}_{2}$ layers," Opt. Mater., vol. 17, no. 1-2, pp. 125-129, Jun. 2001. 